\title{
Kinetic Analysis of Glycoprotein-Lectin Interactions by Label-Free Internal Reflection Ellipsometry
}

\author{
David W. Ralin • Shane C. Dultz • Judd E. Silver • \\ Jeffrey C. Travis • Majlinda Kullolli • \\ William S. Hancock • Marina Hincapie
}

Published online: 18 July 2008

(C) Humana Press 2008

\begin{abstract}
Introduction Glycoproteomics is undergoing rapid development, largely as a result of advances in technologies for isolating glycoproteins and analyzing glycan structures. However, given the number and diversity of glycans, there is need for new technologies that can more rapidly provide differential carbohydrate-protein structural information on a large scale. We describe a new microarray platform based on a label-free imaging ellipsometry technique, which permits simultaneous detection of multiple glycoproteinlectin interactions without the need for reporter labels, while still providing high throughput kinetic information at much lower cost. Our results demonstrate the utility of

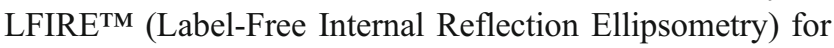
the rapid kinetic screening of carbohydrate-lectin recognition. The technology was also used to evaluate the benefits of the lectin immobilization format using multi-lectin affinity chromatography (M-LAC) to capture glycoproteins (with enhanced binding strength or avidity) from biological samples. Using a printed panel of lectins, singly or in combination, we examined the binding characteristics of standard glycoproteins.
\end{abstract}

David W. Ralin and Shane C. Dultz contributed equally to this work.

D. W. Ralin $(\bowtie) \cdot$ S. C. Dultz · J. E. Silver J. C. Travis

Maven Biotechnologies, LLC,

145 N. Sierra Madre Blvd. Su. 3,

Pasadena, CA 91107, USA

e-mail: dralin@mavenbiotech.com

M. Kullolli · W. S. Hancock • M. Hincapie

Department of Chemistry and Chemical Biology,

Northeastern University,

Boston, MA 02115, USA
Results and Discussion Using kinetic measurements, it was observed that the binding strength of lectins to carbohydrates is enhanced using a multi-lectin strategy, suggesting that improved selectivity and specificity can lead to increased functional avidity. The data presented confirm that this label-free technology can be used to effectively screen single or combinations of lectins. Furthermore, the combination of LFIRETM and M-LAC may permit more rapid and sensitive identification of novel biomarkers based on carbohydrate changes in glycoproteins, and lead to a better understanding of the connections of glycan function in cellular mechanisms of health and disease.

Keywords Lectins - Glycoproteome . Imaging ellipsometry · Label-free · Glycoproteins . Internal reflection ellipsometry $\cdot$ Multiplexing $\cdot$ Kinetics

\section{Introduction}

Glycosylation of proteins is a nearly ubiquitous posttranslational modification observed in eukaryotic organisms. It is estimated that roughly half of the mammalian proteome consists of glycoproteins [1]. Glycosylation plays a fundamental role in a diverse set of biological processes such as immune response, cellular regulation, and cell signaling [2]. Alterations in glycosylation patterns have been linked to the progression of several diseases, most notably cancer [3, 4].

In recognition of the importance of glycosylation, many proteomics researchers have turned their attention to the glycoproteome in search of markers for disease diagnosis and treatment. These include candidate biomarkers that change in either the abundance of the glycoprotein or the glycan structure itself, or both. However, the study of 
glycosylation is a challenging undertaking; this can partly be attributed to the complexity of glycan structures. Glycans can exist in highly heterogeneous forms, with a variety of possible structural configurations and linkage types; they are present as $\mathrm{N}$ - or $\mathrm{O}$-linked and can occupy several sites on a glycoprotein. Several methodologies are available to characterize glycostructures, such as those based on anion exchange separation of fluorescently tagged glycans [5], mass spectrometry using nano-LC coupled to a linear ion trap Fourier transform mass spectrometer (nanoLC-LTQ/FTMS) [6], and MALDI-TOF [7]. In addition, carbohydrate-protein interactions have typically been studied using kinetic measurements by surface plasmon resonance (SPR) $[8,9]$ and frontal affinity chromatography using lectins [10]. Previously, methods focused on carbohydrate arrays, where the low-affinity lectin-carbohydrate interactions are much simpler and easier to model but do not allow researchers to directly examine changes in glycosylation of proteins [11]. In the past few years, lectin microarrays have become increasingly popular. Kuno et. al. reported using a new microarray procedure based on evanescent-field-excited fluorescence detection of lectin microarrays as a strategy for glycan profiling to obtain kinetic information in a multiplexed fashion [12]. Technologies capable of analyzing multiple lectin-glycoprotein interactions in parallel in a single experiment and in a highthroughput manner offer increased utility, as reported previously $[8,11,12]$. Such technologies can be utilized to efficiently study disease-associated glycosylation changes on a proteome scale to further accelerate the quest for "glyco-markers" of clinical value.

Lectins comprise a special family of carbohydratebinding proteins distributed widely in the plant and animal kingdom. After a boom in lectin discovery and characterization in the 1970s, many lectins became commercially available for research purposes [13]. Lectins have been essential for the understanding of carbohydrate-based biological recognition. The use of lectin affinity chromatography has gained popularity in glycoproteomics as a way to simplify the sample before analysis, to enrich specific glycoprotein content in blood samples. In addition, the differential specificity of lectins allows specific populations of glycoproteins to be targeted and may permit changes in glycosylation to be detected. The wide variety of lectins available commercially enables many different subsets of glycans to be investigated. Technologies such as the multi-lectin affinity chromatography (M-LAC) [14] or serial lectin affinity chromatography (S-LAC) [15] have been both used for interrogating the glycoproteomes of human plasma, serum, urine, and cellular lysates. Alternatively, lectin or carbohydrate arrays have also been introduced to study changes in the glycoproteome. A significant benefit of array technologies is that they require very little sample, and a wide spectrum of ligand interactions may be screened rapidly in parallel format.

We (W.H., M.H., M.K.) developed the concept of MLAC or multi-lectin affinity chromatography as a way of improving the specificity/selectivity of glycoproteins isolated from complex biological specimens. We have demonstrated previously $[16,17]$ that the M-LAC column has excellent enrichment capabilities for glycoproteins from human plasma or serum. This technology provides an approach for detecting up or down regulation of glycosylated and non-glycosylated proteins from the same sample. The advantage of M-LAC over single or serial lectin capture may lie in its more complete single-step capture and enrichment of low level glycoproteins from complex samples, such as serum or plasma. We also have demonstrated that M-LAC can be used to monitor changes in protein glycosylation. This is achieved by step-wise elution of glycoproteins using three different sugar displacers specific for each lectin in the M-LAC column. For example, it has been demonstrated that the treatment of transferrin with neuraminidase to remove sialic acid causes a shift in distribution of transferrin isoforms between fractions eluted with three different displacers [18].

The combination of specificities of the lectins as found in the M-LAC column potentially can lead to a functional advantage due to multi-valency and an increase in binding strength over single lectins. It would be advantageous, however, to have a procedure for efficiently optimizing the type and ratio of lectins used in a specific sample set. In this report, we used a prototype imaging instrument (LFIRETM) based on the principles of ellipsometry, a widely used and versatile optical technique for measuring the thickness and optical parameters of ultra-thin films, which can be calibrated directly to the amount of material bound on a wide range of surfaces. The theory of ellipsometry is discussed extensively $[19,20]$ and will not be addressed in detail here. Although the underlying theory of ellipsometry has been understood for over a century [21], it was not until the 1960s that ellipsometers were used routinely in the early computer industry to characterize the growth of thin metallic and semi-conductor films. In the life sciences, there were a few pioneering works using ellipsometry for immunological studies and for measuring the kinetics of protein adsorption. A review of these works from 1972 [22] demonstrated the use of ellipsometry in only a small number of applications. Over a decade later, some of the first quantitative comparisons, between ellipsometry and radiolabeling techniques, of the surface mass density of adsorbed layers of proteins were demonstrated [23]. During the 1990s, imaging ellipsometry and imaging SPR had been developed and used to study the film thickness of biological layers [24-28]. Whereas imaging SPR focuses on reflected light intensity, LFIRE ${ }^{\mathrm{TM}}$ is characterized by the rapid time- 
resolved detection of polarization changes of a reflected monochromatic light beam due to interactions with biomolecules near a glass-liquid interface. The resultant measured phase shift between s-polarized and p-polarized light is more sensitive to adsorbed protein layers than the reflected light intensity signal from imaging SPR. In addition, SPR imaging requires a metallic layer such as gold, which limits the spatial resolution of images due to the spatial extent of the excited surface plasmons [29]. The LFIRETM measurements are performed in situ with high spatial resolution, so that the changes in thickness and refractive index due to specific binding events within the array may be measured. Data is acquired several times per minute so kinetics may be determined for a high number of targets in parallel in both fluid cell and wellplate formats.

The LFIRETM technology was used as a proof of concept to investigate the interaction and kinetics of standard glycoproteins with single and combinations of lectins typically used in the M-LAC format. It was determined that this approach could efficiently measure the binding kinetics of both single and combinations of lectins. The ability to perform multiplexed real-time measurement of binding kinetics adds a new dimension to the field of glycomics by allowing the analyst to probe multiple samples and combinations of lectins to interrogate carbohydrate-lectin interactions of clinical relevance. Post-translational changes in the carbohydrate structure resulting in either trimming or addition of sugars will affect the binding kinetics, a measurement that can be further used to identify and characterize new biomarkers for clinical applications. This will have broad impact in the study of diseases such as cancer, where surface, transport, and secreted proteins all show significant changes in glycosylation.

In this work, we describe the development of a new technology platform (LFIRETM) based on the principles of ellipsometry. We applied it to lectin microarrays and demonstrate that this technology can be a useful tool for quantitative and qualitative analysis of complex kinetic interactions between various combinations of lectins and different carbohydrate motifs. Furthermore, the combination of M-LAC and LFIRETM technologies can be very powerful for high throughput ligand selection and/or characterization of glycoprotein biomarkers that can be isolated from patients with defined diseases.

\section{Materials and Methods}

\section{Lectin Array Construction}

Lectins concanavalin A (ConA), wheat germ agglutinin (WGA), and jacalin supplied by Vector Labs (Burlingame,
CA, USA) were reconstituted in M-LAC binding buffer (25 mM Tris, $\mathrm{pH} 7.4,0.5 \mathrm{M}$ sodium chloride, $1 \mathrm{mM}$ $\mathrm{MnCl}_{2}, 1 \mathrm{mM} \mathrm{CaCl}$, and $0.05 \%$ sodium azide) for storage. To remove Tris, the samples were dialyzed into $50 \mathrm{mM}$ 4-2-hydroxyethyl-1-piperazineethanesulfonic acid-buffered saline, $0.005 \%$ Tween 20 (HBST), supplemented with $1 \mathrm{mM}$ manganese chloride and calcium chloride immediately before printing samples. All lectins were diluted to $0.667 \mathrm{mg}$ protein per milliliter in phosphate-buffered saline (PBS), $\mathrm{pH} 7.5$, for printing on high-sensitivity LFIRETM optical slide substrates, which include a proprietary optical coating (Maven Biotechnologies) under a custom polyethylene glycol (PEG)-based hydrogel $N$-hydroxysuccinimide ester (NHS)-terminated surface chemistry (Accelr8 Technology Corporation, Denver, CO, USA). Mono-amine-terminated 5-kDa PEG was obtained from Nektar Therapeutics, Huntsville, AL, USA. Porcine thyroglobulin, hen egg ovalbumin, bovine fetuin, and asialofetuin glycoproteins were supplied by SigmaAldrich, St. Louis, MO. With a printing program developed by Maven, the SpotBot MicroArrayer, and a single Stealth946 pin (Telechem Inc., Sunnyvale, CA, USA), each slide was printed with two identical arrays of 20 materials including lectins and reference spots, four replicates each, $250 \mu \mathrm{m}$ center-to-center, at $50-60 \%$ relative humidity, $25-27^{\circ} \mathrm{C}$, yielding $\sim 100-\mu \mathrm{m}$-diameter spots. A single pin loading for all slides in each batch reduced variation and allowed maximum flexibility in spot location. Microarrays were immediately packaged in moisture-proof bags with silica gel desiccant and stored at $4^{\circ} \mathrm{C}$ until use.

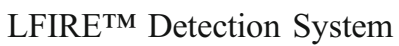

For the current experiments, LFIRE ${ }^{\mathrm{TM}}$ was used in its flowcell configuration to illuminate the surface of a microscope slide, printed with a microarray of lectins, from below with elliptically polarized light from a 1-W, 455-nm LED. Figure 1 is a schematic of the instrument, a modifiedphase-modulated imaging ellipsometer. The light is elliptically polarized by the two optical elements, a polarizer and retarder, which is a quarter-wave plate. The 0.75-in.diameter collimated beam enters a BK7 prism, which is optically coupled with index-matching oil (Cargille, Cedar Grove, NJ, USA) to the underside of the slide. The large incidence angle $\left(\sim 63^{\circ}\right)$ allows the light to be totally internally reflected from the glass/liquid interface, creating an evanescent wave [30] that interacts with refractive index changes within the first $200 \mathrm{~nm}$ of the liquid as glycoproteins approach and are bound by the printed lectins. An image of the reflected beam is projected onto a chargecoupled device camera (Cooke 1600), while the polarizer and retarder are rotated through eight discrete angles called 


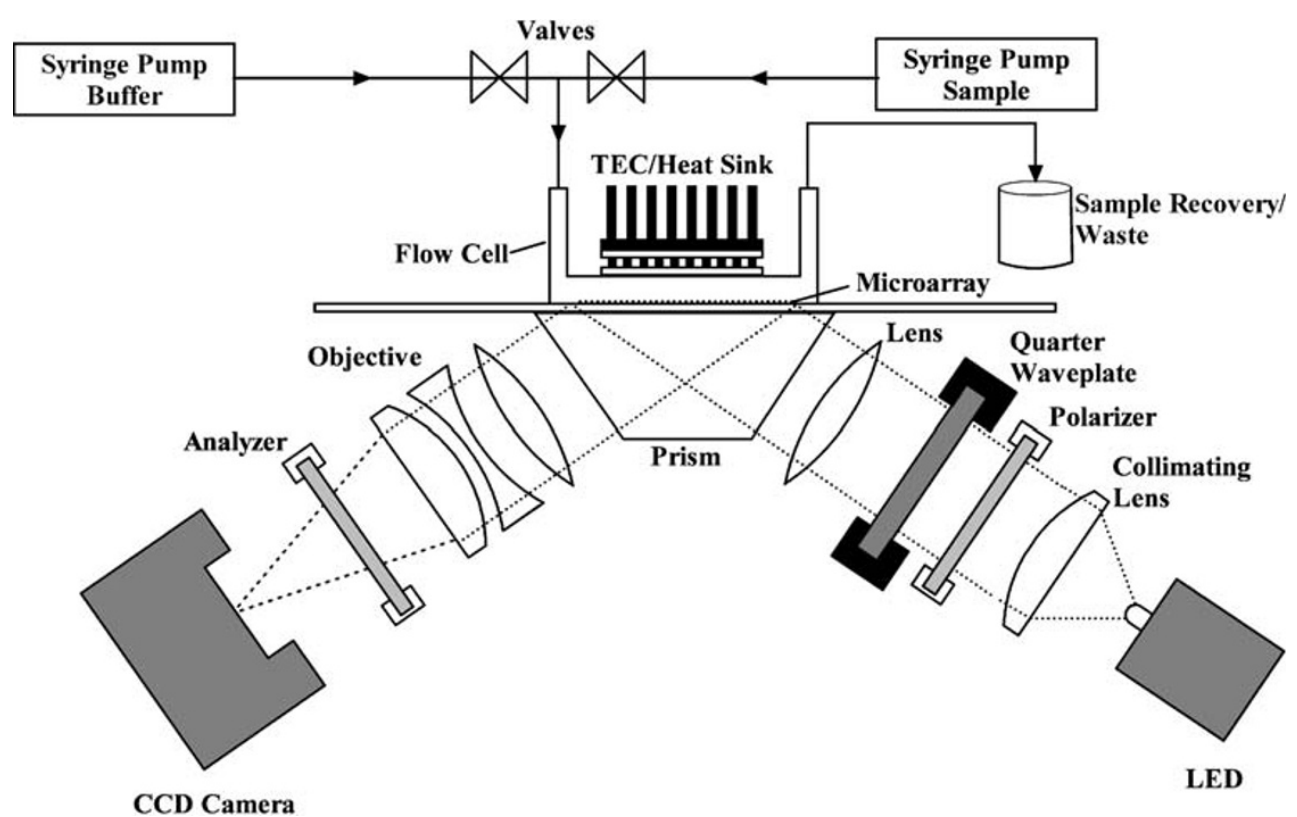

Fig. 1 Schematic drawing of the LFIRE ${ }^{\mathrm{TM}}$ optical setup and associated liquid handling for the flow cell configuration

phases. For each phase in the phase modulation, 15 individual images are averaged together for noise reduction. Those averaged images are then mathematically reconstructed into two final images ( $\mathrm{X}$ and $\mathrm{Y}$ ) every $15 \mathrm{~s}$ for the experimental data. Changes in the Y-image are proportional to the phase shift (retardance) created by the adsorbing molecular layer. This phase shift is in turn proportional to layer thickness or mass density of adsorbed molecules on the surface. The current technology has the sensitivity to detect changes in surface mass density of $3 \mathrm{pg} / \mathrm{mm}^{2}$, which is roughly $25 \mathrm{fg}$ of material in each $100-\mu \mathrm{m}$-diameter microarray spot. This translates to analyte concentrations of 20-40 pM readily detected in a typical antibody-antigen interaction. The instrument is currently capable of capturing the dynamic molecular surface density changes over a maximum area of $12 \times 12 \mathrm{~mm}$ of the substrate slide surface every $30 \mathrm{~s}$ in a flow-cell configuration or with microwells, with 7.4- $\mu \mathrm{m}$ spatial resolution over the entire field of view. Smaller areas can be imaged more rapidly, and all of the data presented in this paper were taken at 15 -s intervals. A second prototype is currently in development to gather microarray data every $3 \mathrm{~s}$ for higher throughput applications requiring 96-well and 384-well plates.

\section{Experimental Setup}

Just before use, lectin microarray slides were washed with an aggressive spray of PBS-T (0.05 M PBS with $0.05 \%$ Tween 20) to remove all loosely bound material, rinsed briefly with distilled water, and dried with a dry nitrogen stream. Slides were attached to LFIRETM disposable twin flow cells and microfluidic interconnect assembly (Fig. 1), optically mated to a BK7 prism with index-matching fluid, enclosed in the LFIRETM TEC environmental control fixture, and inserted in the LFIRE system. The flow cells were filled with M-LAC binding buffer, temperature set to $25 \pm 0.05^{\circ} \mathrm{C}$, and equilibration was obtained before data acquisition. The signal stabilized rapidly in $15 \mathrm{~min}$ due to hydrolysis and quenching of NHS moieties on the surface by the buffer.

To test the surface coating supplier's claim that the surfaces may not require blocking, $50 \mu \mathrm{g} / \mathrm{ml}$ human serum albumin (HSA) was injected; flow was stopped and allowed to incubate for $30 \mathrm{~min}$. HSA did not bind significantly ( $<1 \%$ of a monolayer) to the substrate, lectins, or controls (data not shown). Measuring molecular binding at the sensitivity limits of the instrument requires blocking, normalization to negative control spots such as HSA, or both for this particular surface given that, even within the small panel of glycoproteins, nonspecific binding was observed to the bare substrate but not to the negative control spots.

\section{Lectin/Glycoprotein Kinetic Assay}

The measurement area was set to encompass both arrays, each in its own flow cell, for simultaneous analysis of two glycoproteins. The running buffer for all experiments was M-LAC binding buffer flowing at $25 \mathrm{ul} / \mathrm{min}$. All data was captured as 16-bit TIFFs. After $3 \mathrm{~min}$ of lead-in time to establish the baseline, $10 \mu \mathrm{M}$ glycoprotein samples in the same buffer were injected at a flow rate of $25 \mu \mathrm{l} / \mathrm{min}$, for a net incubation (dwell) time of $4 \mathrm{~min}$. The arrays were rinsed continuously with running buffer only for $15 \mathrm{~min}$ to 
determine off-rates. The final measurements constituted the arbitrary "endpoints" for this study, although most samples still demonstrated some dissociation. All of the glycoproteins except ovalbumin were eluted from the arrays by a 30-min exposure to $20 \mathrm{mM}$ Tris, $0.5 \mathrm{M} \mathrm{NaCl}, 0.17 \mathrm{M}$ methyl-a-D-mannopyranoside, $0.17 \mathrm{M} \mathrm{N}$-acetyl-glucosamine, and $0.27 \mathrm{M}$ galactose, $\mathrm{pH}$ 7.4. Analysis of the images was done using ImageJ v1.34 $\mathrm{s}$ (NIH/public domain), and IgorPro (WaveMetrics, Inc, Lake Oswego, OR, USA) was used for data analysis.

Binding of Thyroglobulin on ConA and M-LAC Columns

Lectins were immobilized onto styrene-divinylbenzene copolymers coated with a cross-linked polyhydroxylated polymer (POROS ${ }^{\mathrm{TM}}$ ). HPLC support using the Schiff base method for covalent immobilization (aldehyde activated media, Applied Biosystems) was used according to the instructions provided by the manufacturer. HPLC columns were packed with either the immobilized Con A or with a mixture of the three individual lectins (ConA, WGA, and JAC) combined in a 1:1:1 (23:8.0:11 mg) molar equivalent ratio to make the M-LAC column. The amount of Con A in the single lectin affinity column was the same as that of the M-LAC support.

Thyroglobulin protein was prepared by dissolving $20 \mathrm{mg}$ in $2 \mathrm{ml}$ of M-LAC binding buffer for a final concentration of $10 \mathrm{mg} / \mathrm{ml} ; 5 \mathrm{mg}$ of the thyroglobulin solution was injected onto each of the ConA and M-LAC columns. To increase the residency time, the protein was loaded slowly $(0.05 \mathrm{ml} / \mathrm{min})$. The flow rate was then increased to $2.5 \mathrm{ml} /$ min. Unbound protein was washed with 10 column volumes (CV) of binding buffer, and the bound protein was eluted with $11 \mathrm{CV}$ of $100 \mathrm{mM}$ acetic acid at $\mathrm{pH} 4.0$. The lectin columns were neutralized with $11 \mathrm{CV}$ of neutralization buffer $(0.5 \mathrm{M}$ Tris, $1 \mathrm{M}$ sodium chloride, and $0.05 \%$ sodium azide, $\mathrm{pH} 7.4$ ) and equilibrated with $10 \mathrm{CV}$ of binding buffer.

\section{Results and Discussion}

A sample image is shown in Fig. 2 of the lectin microarray, printed in quadruplicate, before incubation with glycoproteins. The data is presented in relative ellipsometry units (REUs), the physical meaning of which is described below. Each lectin was printed in quaduplicate with the mean and standard deviation calculated for each set of four replicates. For this particular study, the average of all mean values was 9.5 REU above background, and the average standard deviation was 0.45 REU, considerably larger than the temporal noise of a single spot kinetic trace ( 0.0045 REU). The spot to spot coefficient of variation was in the range

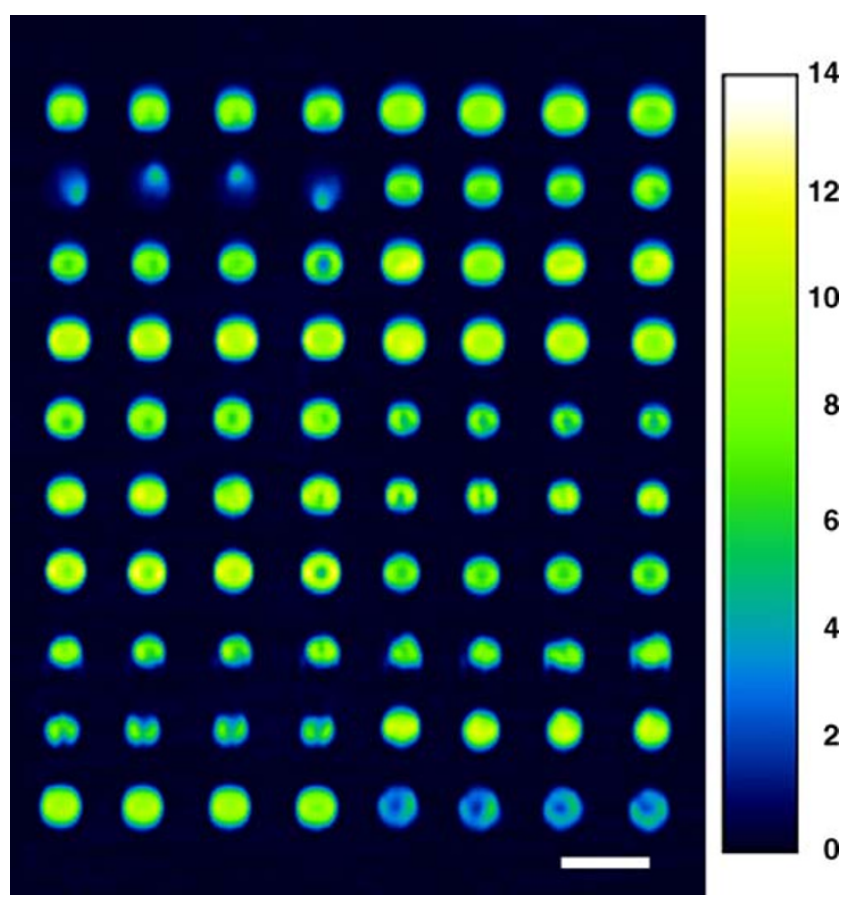

Fig. 2 Initial LFIRE raw image of the lectin microarray in situ and prior to incubation with glycoproteins. Intensity is directly proportional to the surface density of bound protein, allowing evaluation of array quality and correlation with binding measurements. The signal is converted into relative ellipsometry units (REUs), and the color bar inset establishes the relationship between color and signal intensity, which is proportional to the amount of lectin on the surface. Scale bar $=250 \mu \mathrm{m}$

$0.2-11 \%$ for each set of four replicates with an average CV of $4.9 \%$.

Ellipsometry generally gives an average thickness and refractive index value of the protein layer. Feijter's formula is a good approximation between surface mass density and biofilm thickness below.

$M=d \frac{\left(n_{p}-n_{s}\right)}{d n / d c}$

where $M$ is the mass density in $\mathrm{ng} / \mathrm{mm}^{2}, d$ is the thickness of the protein layer in $\mathrm{nm}, n_{p}$ is the index of refraction of the protein layer, $n_{s}$ is the index of the solvent, and $d n / d c$ is a measure of how the index of the protein changes as a function of protein concentration. This value is given by many sources to be $0.182 \mathrm{~cm}^{3} / \mathrm{g}$ [31]. For SPR measurements, an accepted average is that $1 \mathrm{ng} / \mathrm{mm}^{2}$ is equivalent to a 1-nm layer of protein film, which has been confirmed numerous times by SPR [32], quartz-crystal microbalance and AFM measurements. For LFIRETM, $1 \mathrm{ng} / \mathrm{mm}^{2}$ 2.6 REU based on a spotted dilution series of target molecules with concentrations measured by ultraviolet or visible spectroscopy . Figure 2 also depicts a novel feature of LFIRE that permits quality-control assessment of spot 
morphology before initiation of the ligand-binding event. Table 1 describes the relative location of lectins, lectin combinations, and control spots.

Figure 3a through $d$ represents the comparative lectin binding patterns on microarrays of four different glycoproteins. The error bars for this data set was determined to be $\pm 0.1 \mathrm{REU}$ based on the full range of non-specific binding measured by the various negative control spots (data not shown). HSA was chosen as the negative control (assay blank) because those spots were observed to show minimal reactivity with glycoproteins. Thus, the kinetic curves for HSA were subtracted from the kinetic curves for every spot in the array. The data are sorted in ascending order of binding for fetuin, which has three N-linked and three O-linked oligosaccharides, each existing in multiple glycosylation isoforms. The literature suggests that WGA preferentially binds $n$-acetylglucosamine (GlcNAc), which is present in fetuin, but the reduced binding $(\sim 50 \%)$ of asialofetuin suggests that binding is partly dependent on other glycan interactions as well as sialic acid. A test of the specificity of the lectin array is the altered binding order for asialofetuin relative to fetuin, where the increased binding by jacalin is possibly due to increased but not optimal exposure of any of the following: Gal and GalNAc residues or the O-linked glycan core, Galß1-3GalNAc. ConA binds fetuin and asialofetuin weakly, presumably due to minimal exposure of mannose, a finding supported by the understanding of fetuin's glycan structure [33, 34] and by surface plasmon resonance studies [35]. Ovalbumin bound weakly to most of the lectins or their combinations and demonstrated high levels of binding to the bare substrate. Relative binding of ovalbumin to WGA and ConA, and the low binding of ovalbumin in general, supports findings in the literature [11, 36]. Thyroglobulin showed a different binding pattern from the other glycoproteins, most notably no binding with jacalin, consistent with the absence of GalNAc residues in porcine thyroglobulin and the unavailability of terminal Gal $\beta$ in the native sialated glycoprotein

Table 1 Lectin microarray printing map, with each lectin or reference spot printed in quadruplicate

\begin{tabular}{ll}
\hline Column 1 & Column 2 \\
\hline Human serum albumin & Protein A/G \\
5 kDa amino-PEG & ConA/WGA/Jac 0.11:0.22:0.33 \\
ConA/WGA/Jac 0.23:0.29:0:0.17 & ConA/WGA/Jac 0.34:0.12:0.17 \\
HSA/ConA/WGA/Jac 0.23:0.29:0:0.17 & HAS/ConA/WGA/Jac 6:1:1:1 \\
ConA/Jacalin & ConA/WGA/Jac \\
ConA-WGA & WGA/Jac \\
Jacalin & UEA \\
WGA & SNA \\
ConA & PSA \\
Human serum albumin & BPL \\
\hline
\end{tabular}
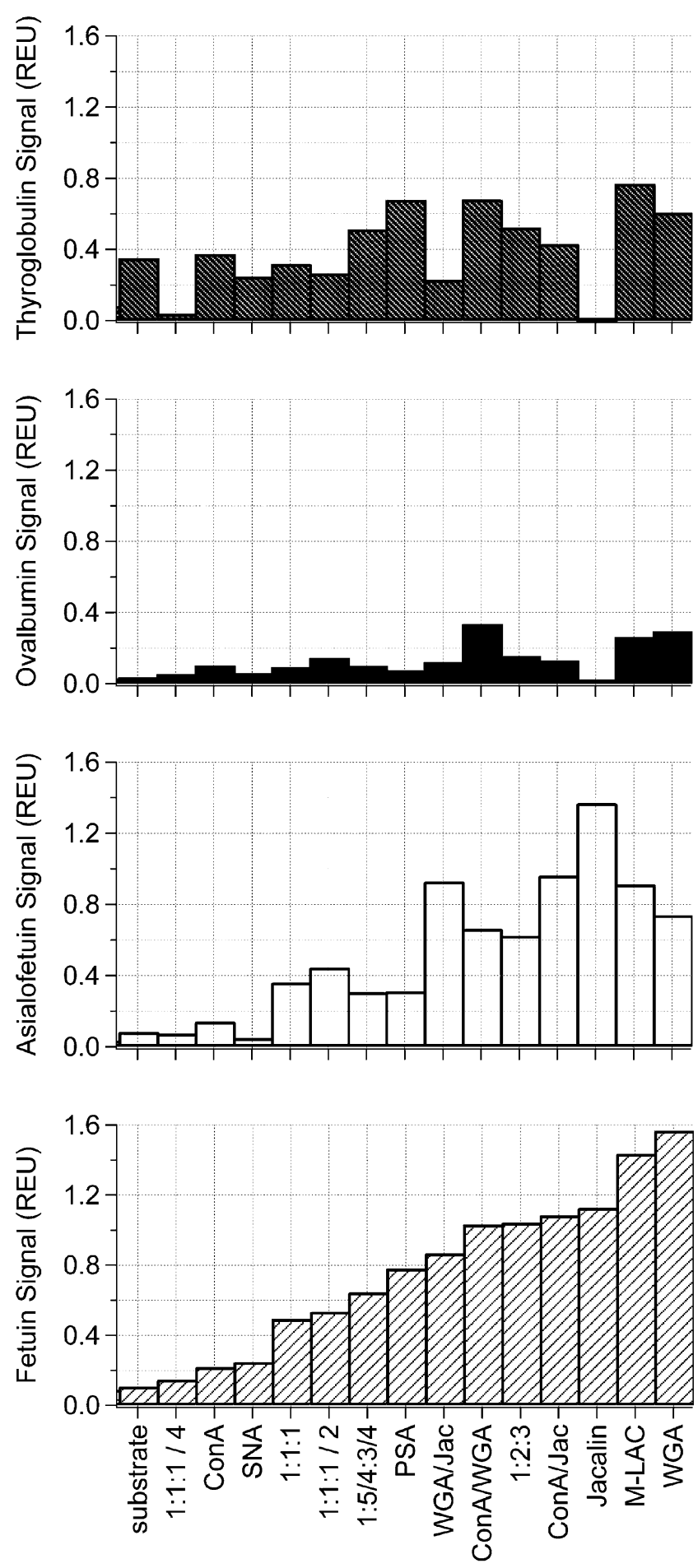

Fig. 3 a-d Binding of four glycoproteins to 14 lectins or lectin combinations after 4-min incubation and $15 \mathrm{~min}$ rinse with running buffer to remove non-specific binding. The lectins are arranged in ascending order in terms of the binding to fetuin, and the same order was repeated for the remaining graphs. The substrate represents areas of the surface not containing lectin and is a measure of non-specific binding. Mixtures are given by three numbers separated by colons and represent the concentration ratio of $\mathrm{Con} \mathrm{A} / \mathrm{WGA} / \mathrm{jacalin}$ within each microarray spot normalized to ConA concentration in milligrams per milliliter. M-LAC is an equimolar ratio of the three lectins 
[37]. Thyroglobulin also bound to the substrate, indicating that studies with glycoproteins require physical blocking of the substrate. Further work is necessary to devise blocking strategies that do not interfere with glycan binding, as common blocking agents such as casein and bovine serum albumin could introduce artifacts. Jacalin's high binding of asialofetuin relative to WGA and ConA has been demonstrated previously with lectin arrays detected by total internal reflection fluorescence scanning [12]. The observation that the binary combination of jacalin and WGA binds asialofetuin equivalently to the binary combination of jacalin and ConA is novel.

Figure 4a through $\mathrm{d}$ shows the differential images of representative lectin spots in the arrays for each of the four glycoproteins tested. Each pair of columns can be matched with the spot ID pattern in Table 1. The brightness of the spots is directly related to the amount of glycoprotein bound and is quantitated by LFIRETM. Clearly, each glycoprotein yields a distinctly different spot pattern, even between fetuin and asialofetuin, which differ only in the presence or absence of terminal sialic acids. Qualitatively, the data suggests that lectin fingerprints could be used for rapid detection of new clinically significant biomarkers in less than $5 \mathrm{~min}$ for glycoprotein concentrations in the low micromolar range.

Figure 5 depicts kinetic curves chosen from two of the lectin spots, each interacting with two of the glycoprotein

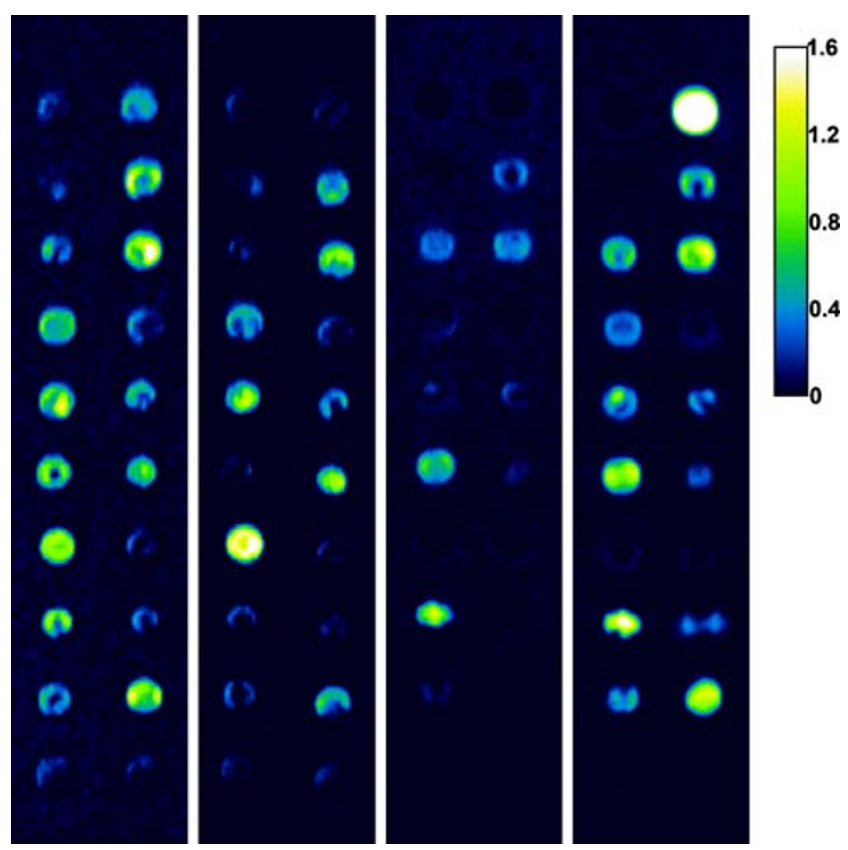

Fig. 4 a-d Differential (endpoint minus baseline) image of columns 4 and 5 from Fig. 2, so as to show one spot of each lectin. a Fetuin, b asialofetuin, $\mathbf{c}$ ovalbumin, and $\mathbf{d}$ thyroglobulin, each after 4-min incubation and 15 -min washing; scale bar $=250 \mu \mathrm{m}$. The spot ID is shown in Table 1 samples, which represents just four of the total 320 interactions studied. As mentioned above, the glycoproteins were introduced into the flow cells at concentrations of $10 \mu \mathrm{M}$ each for a period of $4 \mathrm{~min}$. Although this is insufficient time for the binding to reach equilibrium, relative on and off rates can still be obtained. Before the curves are analyzed, the curves from negative control spots were subtracted from the data to remove changes in the bulk refractive index of the liquid as it changed from buffer to glycoprotein solution and back to buffer again. Spots of human serum albumin were good negative controls for all four glycoproteins. The REU signal is proportional to surface mass density, just as in an SPR system, and conventional curve fits are done to extract kinetic parameters such as on-rate, $k_{a}$, off-rate, $k_{d}$, and affinity, $K_{A}=k_{a} / k_{d}$, which were given in units of inverse molarity. The signals were analyzed using the method of Karlsson et al. [38], which has been the basis of analysis software for surfacebased molecular interactions for almost two decades. The derivation of Eqs. 2 and 3 is based on the simple 1:1 Langmuir interaction model $(A+B \Leftrightarrow A B)$, constraining the kinetic rate constants to a single value [39]. Although the complexity of the data suggests that a more complex model would be needed for an accurate determination of kinetic constants, increasing the number of fit parameters without understanding the source of interaction complexity (i.e., sample heterogeneity, surface conformational changes, multivalent binding, etc.) can produce confounding results. Using the simplified model, the calculated rate constants in this paper should be considered qualitative. In Figure 5, curves were fit in two separate regions. The adsorption region $(t=0-225 \mathrm{~s})$ was fitted using Eq. 2.

$R(t)=R_{0}+R_{\max }\left(1-e^{\left(-\left(C k_{a}+k_{d}\right) t\right)}\right)$

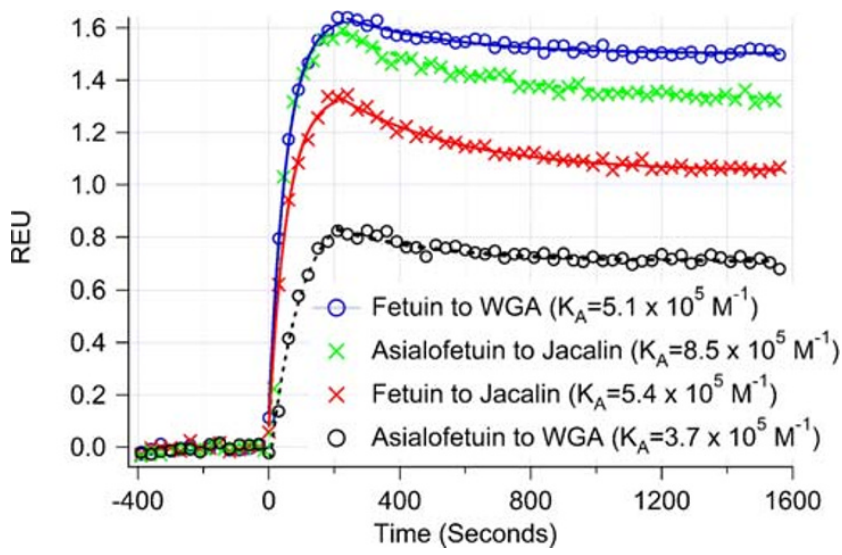

Fig. 5 Kinetic data for fetuin and asialofetuin binding to WGA and jacalin. The fitted curves from the data are used to calculate the affinity, $K_{A}$, equal to the association constant divided by the dissociation constant 


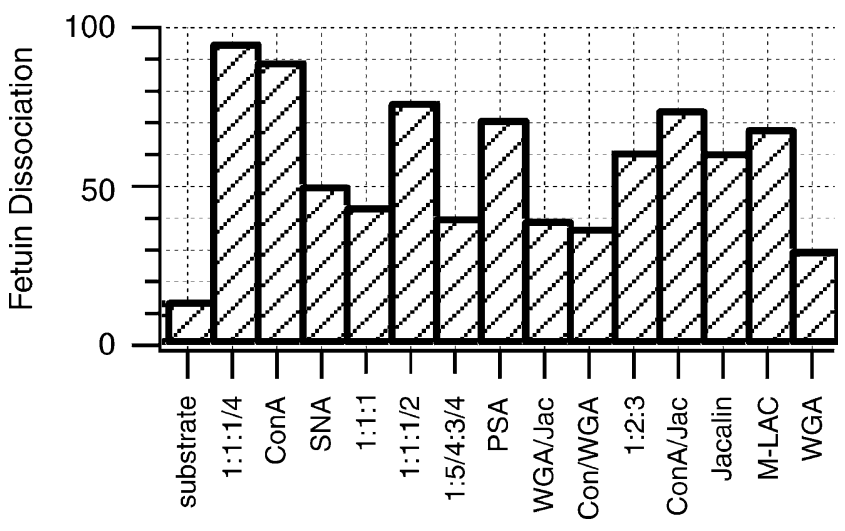

Fig. 6 Dissociation of fetuin from the lectins after introducing $20 \mathrm{mM}$ Tris, $0.5 \mathrm{M} \mathrm{NaCl}, 0.17 \mathrm{M}$ methyl-a-D-mannopyranoside, $0.17 \mathrm{M} \mathrm{N}$ acetyl-glucosamine, and $0.27 \mathrm{M}$ galactose, $\mathrm{pH} 7.4$ for $30 \mathrm{~min}$. The amount of fetuin leaving the surface is compared to the original amount adsorbed

where $R_{0}$ is a constant, $R_{\max }$ is the maximum equilibrium binding at high glycoprotein concentration, $C$ is the concentration of fetuin or asialofetuin during the reaction, $k_{a}$ is the adsorption coefficient in units of $\mathrm{M}^{-1} \mathrm{~s}^{-1}$, and $k_{d}$ is the desorption coefficient in units of $\mathrm{s}^{-1}$. The desorption region $(t>225 \mathrm{~s})$ was fit using Eq. 3.

$R(t)=C+A e^{\left(-k_{d} t\right)}$
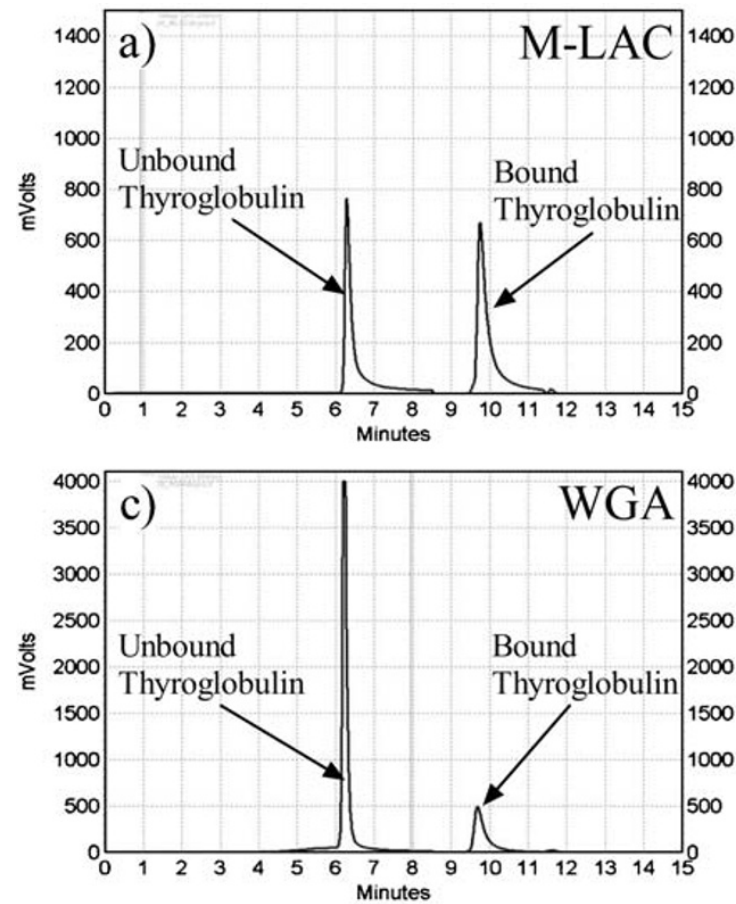

Fig. 7 a-c Affinity chromatography of thyroglobulin on M-LAC, ConA, and WGA, respectively. Chromatographic conditions are described in "Materials and Methods." Bound thyroglobulin was eluted with solvent B (100 mM acetic acid, $\mathrm{pH} 4.0)$, and protein elution was monitored at $280 \mathrm{~nm}$. The quantitation was performed using peak area with $1.6 \%, 21 \%$, and $55 \%$ bound to ConA, WGA, and M-LAC, respectively. d Kinetic data run on LFIRETM showing the
The chi-square value was determined for each curve to determine the quality of the fit. The largest value was 95 , which should be compared to the number of data points in the fitting function, 200 in this analysis. As long as the chisquare value is lower than the number of data points, this can be considered a good fit [40]. The low chi-square value of the fits demonstrates that conventional kinetic analysis can be applied to lectin microarrays using LFIRE technology in a highly multiplexed fashion. The affinity values in Fig. 5 were average values calculated from each of four replicate spots. To get some sense of the variability, affinities were calculated for individual spots showing a range of $\pm 7-8 \%$ about the mean for each interaction. Thus, the calculated affinity for fetuin to WGA is $5.1 \times 10^{5} \pm 0.4 \times$ $10^{5} \mathrm{M}^{-1}$. The variability for asialofetuin to jacalin was $\pm 0.7 \times 10^{5} \mathrm{M}^{-1}$; for fetuin to jacalin, $\pm 0.4 \times 10^{5} \mathrm{M}^{-1}$; and finally, for asialofetuin to WGA, $\pm 0.3 \times 10^{5} \mathrm{M}^{-1}$. Thus, in this experiment, the affinities between fetuin and WGA versus jacalin were too close to distinguish based on kinetics, although substantially, more fetuin was bound to WGA. In the case of asialofetuin, the affinity for jacalin was clearly higher compared to WGA. This difference suggests that asialofetuin, produced by desialation of fetuin, may expose more galactose residues that are recognized by jacalin. The reproducibility of microarray data is based on a
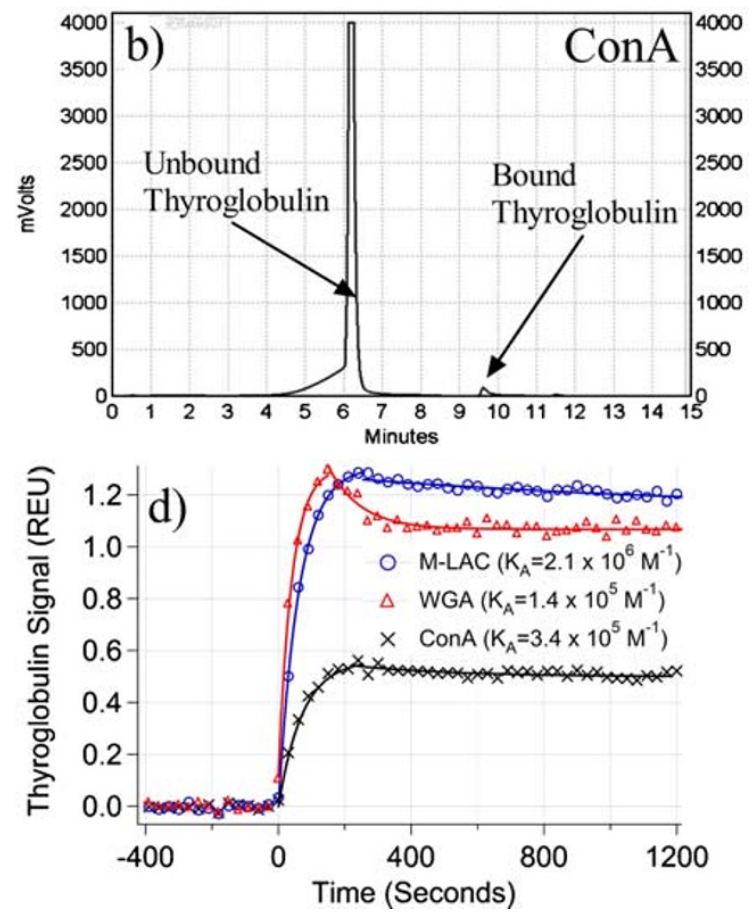

interaction of circulating thyroglobulin with an immobilized spot containing equimolar concentrations of $\mathrm{ConA} / \mathrm{WGA} / \mathrm{jacalin}$ compared to spots containing single lectins, ConA, and WGA. The total binding to the mixed lectin spot is more than twice the amount of binding to ConA. Kinetic fit results show the binding affinity about six times higher for the mixed lectin interaction over ConA and over an order of magnitude over WGA 
number of factors including surface chemistry, spot uniformity, good negative controls, quality of the sample, etc. It is the intention of the authors to show the capabilities for screening lectin-carbohydrate interactions giving semiquantitative results. Although measured affinities are reproducible within 5\% with LFIRE for many interactions such as antibody-antigen binding, more experimental controls need to be in place to measure affinity values for the relatively weak lectin interactions with this degree of precision.

As an example of the data obtained in the study, Fig. 6 shows the dissociation of fetuin from the lectin spots in the presence of a cocktail of monosaccharides. Depending on the lectin composition of the spot, between $30 \%$ and $95 \%$ of the fetuin eluted after a 30-min exposure to the cocktail, far less than expected. Incomplete dissociation may be due to multivalency effects, and a higher concentration of saccharide inhibitor, other than the one used in these studies, may be required to effectively elute the bound glycoprotein. An extensive series of saccharide inhibition studies would help clarify these results. Inspection of the dissociation of the fetuin from the single and paired lectins yielded a consistent relation. In ascending order, the dissocations are WGA $<\mathrm{WGA}+$ jacalin $<$ jacalin + Con A $<$ Con A. Such information could aid the design of chromatographic systems for separating glycoproteins on columns of immobilized lectins.

We examined the binding affinity of porcine thyroglobulin using single (Con A and WGA) or combinations of lectins (M-LAC). This glycoprotein contains approximately $10 \%$ carbohydrate, present as high mannose and complextype oligosaccharides, and thus, has affinity toward ConA and WGA lectins. Lectin affinity chromatography was performed as described in materials and methods. The results are shown in Fig. $7 \mathrm{a}-\mathrm{c}$ and indicate that the amount of thyroglobulin bound to M-LAC (55\%) is significantly higher than that of Con A (1.6\%) or WGA $(21 \%)$. The increased binding observed with the M-LAC is likely due to multi-site attachment between the glycoprotein and the different lectins. Lectin-carbohydrate interactions are relatively weak $\left(10^{2}-10^{6} \mathrm{M}^{-1}\right)$; however, in vivo, lectins exhibit high affinity and specificity for glycoproteins on the surface of cells [41]. It has been postulated that multiple protein-carbohydrate interactions act synergistically to bring about avidity and specificity [42]. To determine if the combination of lectins leads to stronger binding due to multivalent interaction, we measured the binding kinetics to single and multi-lectins.

The kinetic measurements are shown in Fig. $7 \mathrm{~d}$ and were performed under identical experimental conditions as the data shown in Fig. 5. The binding affinity of thyroglobulin for the mixture of lectins $\left(K_{a}=2.1 \times 10^{6} \mathrm{M}^{-1}\right)$ was found to be about six and 15 times higher over ConA (3.4× $\left.10^{5} \mathrm{M}^{-1}\right)$ and WGA $\left(1.4 \times 10^{5} \mathrm{M}^{-1}\right)$, respectively. Although it is not the scope of this paper to define carbohydratelectin multivalent biochemistry, kinetic analysis using multiple and single lectins can greatly aid in the selection of lectin combinations to enrich low-level glycoprotein markers in complex clinical samples such as plasma or serum. In ongoing further studies, we are using LFIRETM imaging ellipsometry as a complementary measurement tool to multi-lectin affinity chromatography for glycoprotein analyses. The combination of both technologies can be used to reveal subtle differences in glycan-binding specificities of closely related lectins or lectin combinations. For this purpose, the microarray format is particularly suitable for studying a single probe's interaction with a large number of targets. Hence, this methodology permits detection of the binding of a specific glycoprotein to a panel of lectins in a multiplex format to monitor subtle changes in glycosylation associated with disease. This can be very relevant in the field of clinical glycoproteomics for the identification and/or validation of "glyco-markers" in diseases such as cancer, which are typically associated with alterations in the amount and type of glycan content. Future experiments are planned to study the structure-function relationships of carbohydrate moieties especially in the discovery of novel clinically relevant biomarkers.

In conclusion, the data suggests that LFIRE ${ }^{\mathrm{TM}}$ technology can be a useful tool for quantitative and qualitative analysis of complex kinetic interactions between various combinations of lectins and glycoproteins. Moreover, by obviating the need for labels, analyses can be performed more economically and in parallel using lectin microarrays. For discovery of potentially significant new biomarkers, the powerful combination of M-LAC and LFIRETM offers the possibility of analyzing lectin-glycoprotein "fingerprints" or patterns associated with disease occurrence, stage, and/or response to drugs.

Acknowledgment Some of the authors (Kullolli, Hancock, and Hincapie) wish to acknowledge support from the National Cancer Institute for grant U01 CA128427-01, "Glycan Markers for the Early Detection of Breast Cancer.”

\section{References}

1. Apweiler R, Hermjakob H, Sharon N. On the frequency of protein glycosylation, as deduced from analysis of the SWISS-PROT database. Biochim Biophys Acta 1999;1473(1):4-8.

2. Opdenakker G, Rudd PM, Ponting CP, Dwek RA. Concepts and principles of glycobiology. FASEB J 1993;7:1330-7.

3. Dube DH, Bertozzi CR. Glycans in cancer and inflammationpotential for therapeutics and diagnostics. Nat Rev Drug Discov 2005;4(6):477-88.

4. Dennis JW, Granovsky M, Warren CE. Glycoprotein glycosylation and cancer progression. Biochim Biophys Acta 1999;1473 (1):21-34. 
5. Yuen C-T, Gee CK, Jones C. High-performance liquid chromatographic profiling of fluorescent labelled N-glycans on glycoproteins. Biomed Chromatogr 2002;16(4):247-54.

6. Wang Y, Wu S-L, Hancock WS. Approaches to the study of Nlinked glycoproteins in human plasma using lectin affinity chromatography and nano-HPLC coupled to electrospray linear ion trap-Fourier transform mass spectrometry. Glycobiology 2006;16(6):514-23.

7. Novotny MV, Mechref Y. New hyphenated methodologies in high-sensitivity glycoprotein analysis. J Sep Sci 2005;28 (15):1956-68

8. Smith EA, Thomas WD, Kiessling LL, Corn RM. Surface plasmon resonance imaging studies of protein-carbohydrate interactions. J Am Chem Soc 2003;125:6140-8.

9. Duverger E, Frison N, Roche AC, Monsigny M. Carbohydratelectin interactions assessed by surface plasmon resonance. Biochimie 2003;85(1):167-79.

10. Tateno H, Nakamura-Tsuruta S, Hirabayashi J. Frontal affinity chromatography: sugar-protein interactions. Nature Protocols 2007;2(10):2529-37.

11. Pilobello KT, Krishnamoorthy L, Slawek D, Mahal LK. Development of a lectin microarray for the rapid analysis of protein glycopatterns. Chem BioChem 2005;6:985-9.

12. Kuno A, Uchiyama N, Koseki-Kuno S, et al. Evanescent-field fluorescence-assisted lectin microarray. Nature Methods 2005;2 (11):851-6.

13. Sharon N, Lis H. History of lectins: from hemagglutinins to biological recognition molecules. Glycobiology 2004;14 (11):53R-62R.

14. Yang Z, Hancock WS. Approach to the comprehensive analysis of glycoproteins isolated from human serum using a multi-lectin affinity column. J Chromatgr A 2004;1053:79-88.

15. Durham M, Regnier FE. Targeted glycoproteomics: serial lectin affinity chromatography in the selection of $O$-glycosylation sites on proteins from the human blood proteome. J Chromatogr A 2006;1132(1-2):165-73.

16. Yang Z, Harris LE, Palmer-Toy DE, Hancock WS. Multilectin affinity chromatography for characterization of multiple glycoprotein biomarker candidates in serum from breast cancer patients. Clin Chem 2006;52:1897-905.

17. Plavina T, Wakeshull E, Hancock WS, Hincapie M. Combination of abundant protein depletion and multi-lectin affinity chromatography (M-LAC) for plasma protein biomarker discovery. J Proteome Res 2007;6(2):662-71.

18. Yang Z, Hancock WS. Monitoring glycosylation pattern changes of glycoproteins using multi-lectin affinity chromatography. J Chromatgr A 2005;1070(1-2):57-64.

19. Azzam RMA, Bashara NM. Ellipsometry and polarized light. Amsterdam: Elsevier; 1987. p. 153-268.

20. Humlicek J. Polarized light and ellipsometry. In: Tompkins HG, Irene EA, editors. Handbook of ellipsometry. New York: William Andrew Publishing; 2005. p. 3-91.

21. Drude PKL. Zur Elektronentheorie der Metalle. Annalen der Physik 1900;1:566-613.

22. Poste G, Moss C. The study of surface reactions in biological systems by ellipsometry. Prog Surf Sci 1972;2:139-232.

23. Jonsson U, Malmqvist M, Ronnberg I. Adsorption of immunoglobulin $\mathrm{G}$, protein $\mathrm{A}$, and fibronectin in the submonolayer region evaluated by a combined study of ellipsometry and radiotracer techniques. J Colloid Interface Sci 1985;103:360-72.

24. Rothenhausler B, Knoll W. Surface-plasmon microscopy. Nature 1988;332:615-7.

25. Liu AH, Wayner PC Jr, Plawsky JL. Image scanning ellipsometry for measuring non-uniform film thickness profiles. Appl Opt 1994;33:1223.

26. Jin G, Tengvall P, Lundstrom I, Arwin H. A biosensor concept based on imaging ellipsometry for visualization of biomolecular interactions. Anal Biochem 1995;232(1):69-72.

27. Noort DV, Rumberg J, Jager EWH, Mandenius CF. Silicon based affinity biochips viewed with imaging ellipsometry. Meas Sci Technol 2000;11:801-8.

28. Venkatasubbarao S, Beaudry N, Zhao Y, Chipman R. Evanescentimaging-ellipsometry-based microarray readers. J Biomed Opt 2006;11(1):14028-37.

29. Berger CEH, Kooyman RPH, Greve J. Surface plasmon propagation near an index step. Opt Commun 1999;167(1-6):183-9.

30. De Fornel F. Evanescent waves from Newtonian optics to atomic optics. Berlin: Springer; 2001.

31. De Feijter JA, Benjamins J, Veer FA. Ellipsometry as a tool to study the ad-sorption of synthetic and biopolymers at the airwater interface. Biopolymers 1978;17:1759-72.

32. Stenberg E, Persson B, Roos H, Urbaniczky CJ. Quantitative determination of surface concentration of protein with surface plasmon resonance using radiolabeled proteins. Colloid Interface Sci 1991;143(2):513.

33. Baenziger JU, Fiete D. Structure of the complex oligosaccharides of fetuin. J Biol Chem 1979;254(3):789-95.

34. Green ED, Adelt G, Baenziger JU, Wilson S, Van Halbeek HJ. The asparagine-linked oligosaccharides on bovine fetuin. Structural analysis of N-glycanase-released oligosaccharides by 500 megahertz 1H NMR spectroscopy. J Biol Chem 1988;263 (34):18253-68.

35. Okazaki T, Hasegawa Y, Shinohara Y, Bhikhabhai R, Kamasaki T. Determination of the interactions between lectins and glycoproteins by surface plasmon resonance. J Mol Recognit 1995; 8:95-9.

36. Ramos MC, Cavada BS, Mazard A, Rouge P. Interaction of Diocleinae lectins with glycoproteins based in surface plasmon resonance. Mem Inst Oswaldo Cruz 2002;97(2):275-9.

37. Nagano CS, Debray H, Nascimento KS, et al. HCA and HML isolated from the red marine algae Hypnea cervicornis and Hypnea musciformis define a novel lectin family. Protein Sci 2005;14:2167-76.

38. Karlsson R, Michaelsson A, Mattson L. Kinetic analysis of monoclonal antibody-antigen interactions with a new biosensor based analytical system. J Immunol Methods 1991;145:22940.

39. Morton TA, Myszka DG, Chaiken IM. Interpreting complex binding kinetics from optical biosensors: a comparison of analysis by linearization, the integrated rate equation, and numerical integration. Anal Biochem 1995;227:176-85.

40. BIAtechnology Handbook, version AB, reprinted 1998.

41. Sharon N. When lectin meets oligosaccharide. Nat Struct Biol 1994;1(12):843-5.

42. Drickamer K. Multiplicity of lectin-carbohydrate interactions. Nat Struct Biol 995;2(6):437-9. 